\title{
Activity of praziquantel on in vitro transformed Schistosoma mansoni sporocysts
}

\author{
ACA Mattos, JR Kusel**, PFP Pimenta*, PMZ Coelho/***/+
}

Laboratório de Esquistossomose *Laboratório de Entomologia Médica, Centro de Pesquisas René-Rachou-Fiocruz, Av. Augusto de Lima 1715, 31190-002 Belo Horizonte, MG, Brasil **Division of Infection and Immunity, IBLS, University of Glasgow, Scotland, UK ***Santa Casa de Misericórdia de Belo Horizonte, Belo Horizonte, MG, Brasil

Praziquantel (PZQ) is effective against all the evolutive phases of Schistosoma mansoni. Infected Biomphalaria glabrata snails have their cercarial shedding interrupted when exposed to PZQ. Using primary in vitro transformed sporocysts, labeled with the probe Hoechst 33258 (indicator of membrane integrity), and lectin of Glycine max (specific for carbohydrate of $\mathrm{N}$-acetylgalactosamine membrane), we evaluated the presence of lysosomes at this evolutive phase of $\mathrm{S}$. mansoni, as well as the influence of $P Z Q$ on these acidic organelles and on the tegument of the sporocyst. Although the sporocyst remained alive, it was observed that there was a marked contraction of its musculature, and there occurred a change in the parasite's structure. Also, the acidic vesicles found in the sporocysts showed a larger delimited area after contact of the parasites with PZQ. Damages to the tegument was also observed, as show a well-marked labeling either with Hoechst 33258 or with lectin of Glycine max after contact of sporocysts with the drug. These results could partially explain the interruption/reduction mechanism of cercarial shedding in snails exposed to $P Z Q$.

Key words: praziquantel - fluorescence - Hoechst 33258 - LysoTracker Red - lectin of Glycine max - sporocysts Schistosoma mansoni

Praziquantel (PZQ) is the drug of choice of the World Health Organization for schistosomiasis treatment (Doenhoff et al. 2002). Nevertheless, the mechanisms of action of this drug against Schistosoma mansoni are not yet precisely clarified until now (Cioli \& Picca-Mattoccia 2003). It is well known that PZQ is effective against all the evolutive phases of the parasite, being more active against cercariae and adult worms (Gonnert \& Andrews 1977). Previous studies in our laboratory, as well as data from the literature, demonstrated that PZQ acts on the intramolluscan phase of Schistosoma interrupting cercarial shedding (Coles 1979, Touassem \& Combes 1986, Yi \& Combes 1987, Coelho et al. 1988, Riley \& Chappell 1990).

Morphological and physiological changes in $S$. mansoni under the effect of PZQ, at different evolutive phases of the parasite's life cycle, have been demonstrated using as a tool fluorescent labels. Oliveira et al. (2006) demonstrated that PZQ causes damages to the tegument of adult worms, and that these lesion sites are markedly labeled by the probe Hoechst 33258 (specific for DNA and being utilized as indicator of membrane integrity). However, the same lesions are not labelled by the probe lectin of Glycine max (specific for $\mathrm{N}$-acetylgalactosamine, a carbohydrate present in the parasite tegument).

In studies using $S$. mansoni, Al-Adhami et al. (2003) reported the presence of acidic compartments, function-

\footnotetext{
$\overline{\text { Financial support: Fiocruz-CNPq }}$

${ }^{+}$Corresponding author: coelhopm@cpqrr.fiocruz.br

Received 25 May 2006

Accepted 26 June 2006
}

ally similar to lysosomes, in schistosomula, and the absence of them in cercariae, by means of the label Lyso Tracker Red, specific for lysosomes of mammal cells (Dietl et al. 1996). In another study, Al-Adhami et al. (2005) observed the presence of large acidic organelles in immature worms (lung stage), but not in adult worms and miracidia. Carneiro-Santos et al. (2001), besides reporting the presence of lysosomes in schistosomula, verified that PZQ interferes with the formation of acidic organelles. Thus, we evaluated in this work the presence of lysosomes in in vitro transformed $S$. mansoni sporocysts, as well as the activity of PZQ on those acidic organelles and on the parasite's tegument, by means of fluorescent labels.

\section{MATERIALS AND METHODS}

In vitro transformation of sporocysts - Infected mice with $S$. mansoni cercariae (LE strain) were sacrificed after 50-day-infection. Their livers were collected and kept in sterile saline $0.85 \%$, containing $10 \%$ antibiotics penicillin/ streptomycin (Sigma Chemical Co., St. Louis, MO, US), at room temperature, for $30 \mathrm{~min}$. Afterwards, the livers were homogenized and washed to obtain miracidia, using sterile materials, according to the technique described by Pellegrino and Siqueira (1968). Miracidia were gathered into conic tubes of $50 \mathrm{ml}$ and maintained on ice for $30 \mathrm{~min}$. The supernatant was discarded and $5 \mathrm{ml}$ liquid with miracidia were kept apart. Miracidia were cultured/transformed in culture medium RPMI-1640 pH 7.4 (Sigma) supplemented with $5 \%(\mathrm{v} / \mathrm{v})$ inactivate fetal bovine serum (FBS) (Gibco Limited, Paisley, Scotland, UK) and $20 \mu \mathrm{g} / \mathrm{ml}$ antibiotic gentamicine in B.O.D., at $26^{\circ} \mathrm{C}$, for $24 \mathrm{~h}$. Cultured medium $(15 \mathrm{ml})$ was stored in bottles of $25 \mathrm{~cm}^{2}$ each, with addition of $1 \mathrm{ml}$ of liquid containing miracidia. After this period, the bottle contents were put into tubes of $50 \mathrm{ml}$, which were maintained at room temperature, for $30 \mathrm{~min}$. Next they were 
just once washed with non-supplemented culture medium for use in different experiments.

Labels/drugs - Different experiments were carried out with sporocysts labeled with $5 \mu 1$ Hoechst 33258 (Sigma) $10 \mathrm{mg} / \mathrm{ml}$ or with $5 \mu \mathrm{l}$ lectin of Glycine max soybean conjugated to rhodamine (Sigma) $1 \mathrm{mg} / \mathrm{ml}$ or with $20 \mu 1$ Lyso Tracker Red (Molecular Probes, Eugene, Ore) stock solution $10 \mu \mathrm{l}(1 \mathrm{mM})$ in $1 \mathrm{ml}$ culture medium. This labeling was performed using an incubator with $5 \% \mathrm{CO}_{2}$, at $37^{\circ} \mathrm{C}$, for $30 \mathrm{~min}$. The sporocysts were exposed to $5 \mu \mathrm{g} / \mathrm{ml}$ and 10 $\mu \mathrm{g} / \mathrm{ml}$ of praziquantel diluted in methanol, under the same preceding conditions, for $15 \mathrm{~min}$. Methanol $(10 \mu \mathrm{l})$ was added to the control group.

Fluorescence - Analysis of fluorescence was performed under fluorescence Zeiss microscope (K-Zess), filer DAPI and documented in Nikon 4.D megapixel for the probe Hoechst 33258. The probes lectin of Glycine max and LysoTracker Red were analyzed by means of a confocal laser microscope (LMS 510) utilizing laser Helio/Neon $543 \mathrm{~nm}$, filter rhodamine.

\section{RESULTS}

Evaluation of $P Z Q$ activity on in vitro transformed sporocysts - The sporocysts were exposed to PZQ and analyzed under optical microscopy aiming at verifying the activity of PZQ on in vitro transformed sporocysts. It was observed that PZQ, irrespective of the concentration utilized (5 or $10 \mu \mathrm{g} / \mathrm{ml})$, causes a marked contraction of the sporocyst, easily detected under microscopy. It is interesting to note that this contraction seems to be directly proportional to drug concentration (Fig. 1). The parasites, even while exhibiting morphological changes, remained alive and in motion.

Evaluation of the effect $P Z Q$ on the membrane of in vitro transformed sporocysts - Two simultaneous experiments were carried out in order to evaluate the effect of PZQ on the membrane of sporocysts. The probe Hoechst 33258 was used in one experiment and the labeled lectin of Glycine max was utilized in the other one. The sporocysts were labelled and later on exposed at different concentrations of the drug. Using the probe Hoechst 33258 it was possible to observe a slight label in the control group (Fig. 2A). Nevertheless, after exposure to PZQ, a marked increase in label could be observed (Fig. 2B, C), thus suggesting damage on the membrane. In relation to lectin of Glycine max, the sporocyst controls (not exposed to the drug) did not present any labeling and could be named negative controls (Fig. 2D). However, the sporocysts put in contact with the drug showed a marked labelling of the membrane, irrespective of the drug concentration utilized, so demonstrating exposition of $\mathrm{N}$-acetylgalactosamina in these sites (Figs 2F-G, 2H-I).

Evaluation of the presence of lysosome-like vesicles in sporocysts, and the influence of PZQ on acidic organelles - The sporocysts exposed to the probe Lyso Tracker Red showed a marked labeling of acidic vesicles (lysosomes), demonstrating for the first time the presence of organelles at that stage of $S$. mansoni life cycle. Besides the presence of organelles, it was possible to verify that sporocysts exposed to PZQ, at 5 and $10 \mu \mathrm{g} / \mathrm{ml}$ concentrations (Figs 3C-D, 3E-F, respectively), presented an altered structure showing a larger area labeled by the probe, when compared with the control sporocysts that were not exposed to the drug (Fig. 3A, B).

\section{DISCUSSION}

The effect of PZQ on the intramolluscan phase of Schistosoma has already been reported in various works (Coles 1979, Touassem \& Combes 1986, Yi \& Combes 1987, Coelho et al. 1988, Riley \& Chappell 1990). In these studies, it was demonstrated that cercarial shedding is interrupted in infected mollusc up to 17 days after treatment with PZQ. After this period, the molluscs shed cercariae again. Further, it was demonstrated, by means of histology, that primary and secondary sporocysts remain intact after contact with PZQ. These authors suggest that PZQ may be effective against sporocysts, but they are able to recover from the drug effect restablishing cercarial shedding.

In our study, using in vitro transformed sporocysts, it was verified that a marked contraction of their muscular layer (Basch \& Di Conza 1974) occurred after contact with the drug irrespective of its concentration. Nevertheless, the parasites remained alive and in motion, thus suggesting that PZQ, at the concentrations used, it was not able to kill them. Among the mechanisms of PZQ action, the muscular contraction is well known. Muscular contrac-
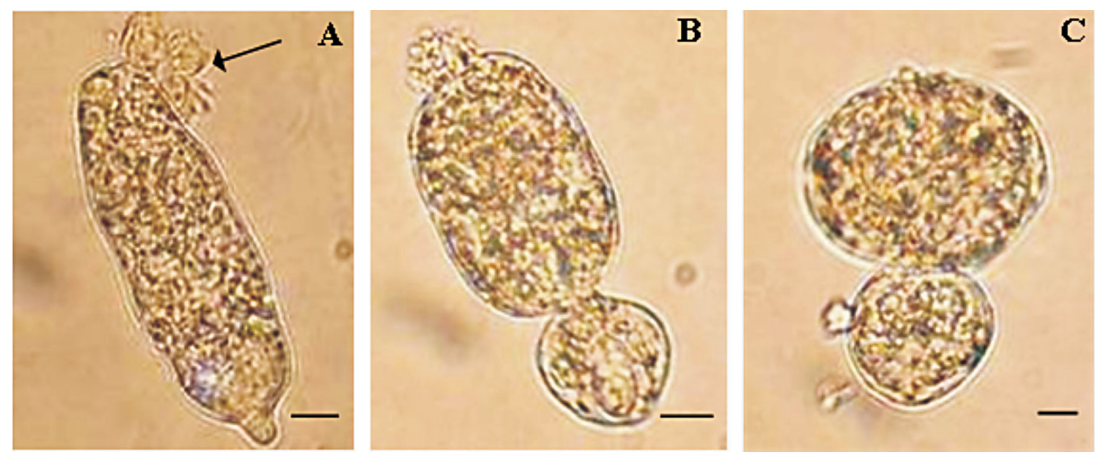

Fig. 1: evaluation of praziquantel (PZQ) activity on in vitro transformed sporocysts. A: sporocyst not exposed to PZQ, normal morphology. The arrow indicates loss of ciliar plate; B: sporocyst exposed to $5 \mu \mathrm{g} / \mathrm{ml} \mathrm{PZQ;} \mathrm{C:} \mathrm{sporocyst} \mathrm{exposed} \mathrm{to10} \mu \mathrm{g} / \mathrm{ml} \mathrm{PZQ}$, marked contraction and change of the parasite's structure. Images obtained under optical microscopy. Bars $=20 \mu \mathrm{m}$. 
tion has been observed in adult worms exposed to PZQ in vitro. Pax et al. (1978) and Liang et al. (2001, 2003) reported the same contraction (as observed in primary sporocysts in our study) in miracidia exposed to PZQ in vitro.

According to Mehlhorn et al. (1981), PZQ is able to cause damage on Schistosoma tegument. By means of an intense labeling of sporocysts with the probe Hoechst
33258 and lectin of Glycine max, it was possible to observe that PZQ is capable of damaging the tegument of sporocysts exposed to the drug. As reported by Oliveira et al. (2006), the probe Hoechst 33258 was able to label damages caused by PZQ in adult worms, but the same event could not be observed when the probe lectin of Glycine max was used. Adult worms are endowed with
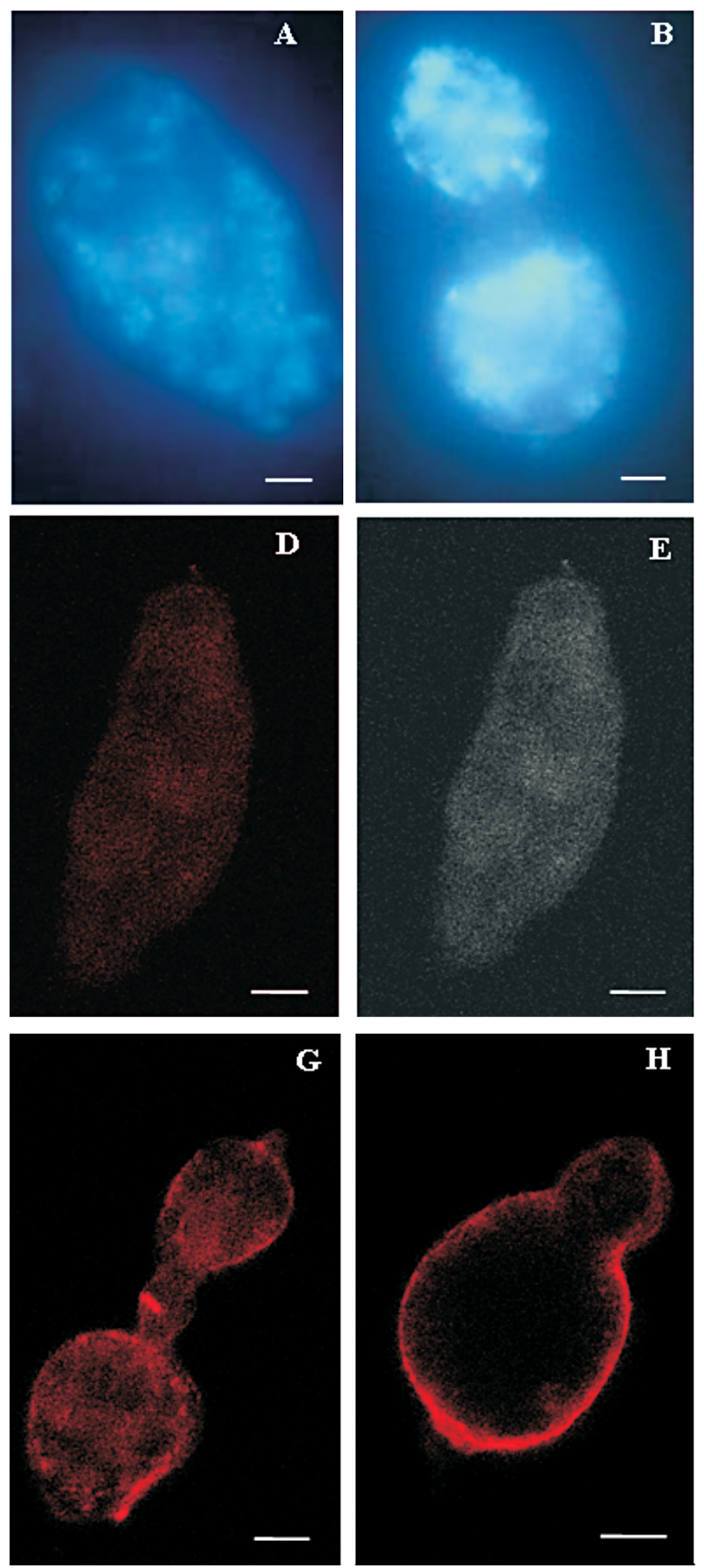
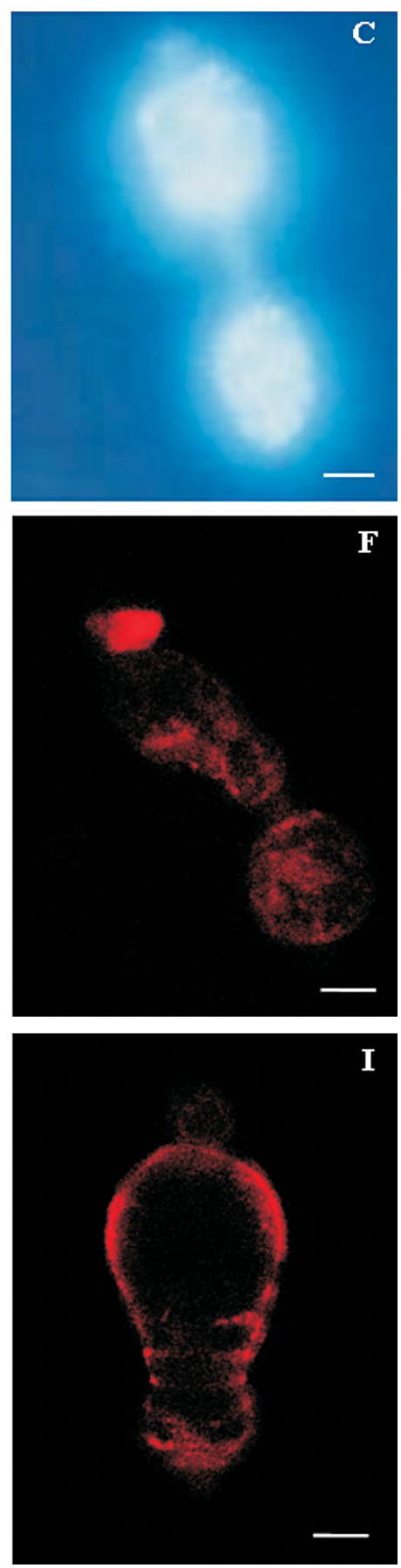

Fig. 2: evaluation of praziquantel (PZQ) activity on the membrane of in vitro transformed sporocyst. A-C: parasites labeled with Hoechst 33258. Images obtained under fluorescence microscopy. A: control sporocyst; B-C: sporocysts exposed to 5 and $10 \mu \mathrm{g} / \mathrm{ml} \mathrm{PZQ}$, respectively. It is possible to observe a slight labeling on the control and an increase of fluorescence on those exposed to the drugs; D-I: sporocysts labeled with lectin of Glycine max; D-E: control parasites; F-G: sporocysts exposed to $5 \mu \mathrm{g} / \mathrm{ml}$ PZQ; H-I: sporocysts exposed to $10 \mu \mathrm{g} / \mathrm{ml} \mathrm{PZQ}$. Labeling with the probes is visible only in the sporocyts exposed to the drugs. Images obtained under confocal laser microscopy. Bars $=20 \mu \mathrm{m}$. 
tegument composed of a thick syncytial cytoplasm overlaid by a double membrane (McLaren 1980). However, these two layers are not observed in sporocysts that show a thin cellular layer with approximately $0.5 \mu \mathrm{m}$ thickness (Smith \& Chernin 1974), wich is possibly more sensitive and even more permeable, when compared with adult worms, being therefore easily damaged.

The probe Hoechst 33258 is utilized as a label for membrane integrity. Thus, it is a very sensitive probe for labeling tegument damages. Any tegument damage, no matter how little it is, can be detected by this probe (Oliveira 2005). This could explain the slight labeling present in the sporocysts not exposed to the drug, whose membrane could be a little damaged throughout the cultivation period in which transformation is occurring. A similar sensitivity was not observed in relation to the probe lectin of Glycine max, since only the sporocysts exposed to PZQ showed an intense labeling of the membrane.

Lysosomes are important compartments involved in cellular digestion (Jans et al. 2004). Carneiro-Santos et al. (2001) demonstrated the presence of acidic organelles similar to lysosomes in schistosomula, as well as the absence of the same organelles in cercariae. These data were confirmed by Al-Adhami et al. (2003, 2005). In this latter study, it was also demonstrated that miracidia do not reveal the presence of acidic vesicles when exposed to LysoTracker Red. The authors suggest that lysosomes could be involved in survival processes and adaptation of schistosomula to their new site in the vertebrate host. This would explain the development of those organelles immediately after transformation of cercariae into schistosomula. The same event could occur during miracidium/sporocyst transformation, since our results demonstrated the presence of acidic organelles in primary sporocysts. Metabolic changes occur during this transformation and this could lead to appearance of acidic vesicles. Probably these acidic vesicles exert a fundamental role in the survival and adaptation of sporocysts to their new site in the invertebrate host. Another event that could occur throughout the transformation period is autophagy, a process utilized by the cells in order to eliminate unnecessary cellular components during cellular differentiation (De Duve \& Wattiaux 1966). In this way, we suggest that during the transformation process, either from cercaria into schistosomulum or miracidium into sporocyst, induction of the autophagy process could occur, leading to development of acidic vesicles.

Our results also showed that PZQ interferes with for-
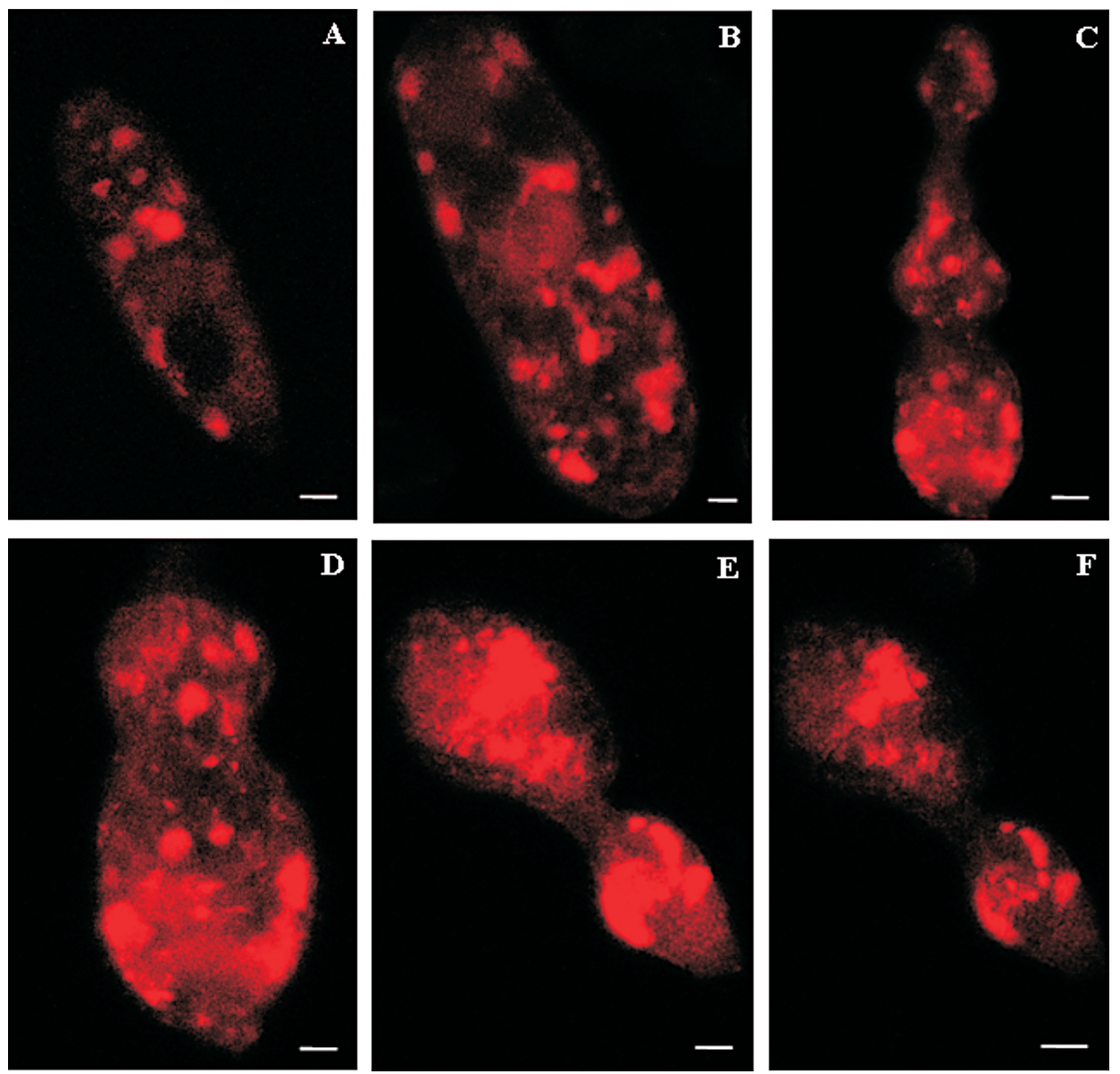

Fig. 3: evaluation of the presence of lysosome-like organelles in sporocyts and the influence of praziquantel (PZQ) on acidic organelles. A-B: sporocysts labeled with $5 \mu 1$ LysoTracker Red and not exposed to praziquantel (PZQ). Presence of some acidic vesicles; C-D: sporocyts labeled with LysoTracker Red and exposed $5 \mu \mathrm{g} / \mathrm{ml}$ PZQ; E-F: sporocysts labeled with LysoTracker Red and exposed to $10 \mu \mathrm{g} /$ $\mathrm{ml} \mathrm{PZQ}$. Presence of the larger labeled area. Images obtained under confocal laser microscopy. Bars: A, C, E, F $=20 \mu \mathrm{m} ; \mathrm{B}, \mathrm{D}=10 \mu \mathrm{m}$. 
mation of acidic organelles. It was possible to observe an increase in the area labeled by LysoTracker Red, after exposure of the parasites to the drug, thus suggesting a more intense activity of those organelles. According to Carneiro-Santos et al. (2001) lysosomes act as intracellular deposits, in order to allow the parasites to get free from the drug. These acidic vesicles could be able to eliminate the drug before the parasite's destruction. This hypothesis is also corroborated in studies carried out by AlAdhami et al. (2005), demonstrating the presence of those acidic compartments in immature worms (lung stage, naturally resistant to PZQ), as well as their absence in adult worms naturally susceptible to PZQ. Besides, autophagy has been described in $S$. mansoni adult worms after drug treatment (Clarkson \& Erasmus 1984).

It followed from these experiments that PZQ is effective against primary sporocysts, causing damages to the tegument and changes in the labeling of the parasite's acidic vesicles. The presence of these organelles in sporocysts is described for the first time in our study. Besides, these results could partially explain the interruption/reduction mechanism of cercarial shedding in snails exposed to PZQ.

\section{ACKNOWLEDGEMENTS}

To Dr Diana Bahia and to Lívia das Graças Avelar for their support related to the technique of in vitro transformed sporocysts; to Nágila Francinete Costa Secundino for her kind assistance; to Tatiana Aguiar Silva and Ana Cristina de Carvalho Botelho for technical support with the analysis of confocal laser microscopy; to Gustavo Ferreira Martins for his collaboration with the format of the photos showed in the text; to Vera Ribeiro for translation from Portuguese; to the technicians of the Mollusc Room, at the Research Center René-RachouFiocruz, for providing the parasites.

\section{REFERENCES}

Al-Adhami BH, Thornhill J, Akhkha A, Doenhoff MJ, Kusel JR 2003. The properties of acidic compartments in developing schistosomula of Schistosoma mansoni. Parasitology 127: 253-264.

Al-Adhami BH, Noble C, Sharaf O, Thornhill J, Doenhoff MJ, Kusel JR 2005. The role of acidic organelles in the development of schistosomula of Schistosoma mansoni and their response to signalling molecules. Parasitology 130: 309322.

Basch PF, DiConza JJ 1974. The miracidium-sporocyst transition in Schistosoma mansoni: surface changes in vitro with ultrastructural correlation. J Parasitol 60: 935-941.

Carneiro-Santos P, Thornhill JA, Doenhoff MJ, Hagan P, Kusel JR 2001. Acidic vesicles of Schistosoma mansoni. Parasitol Res 87: 1001-1006.

Cioli D, Pica-Mattoccia L 2003. Praziquantel. Parasitol Res 90: S3-S9.

Clarkson J, Erasmus DA 1984. Schistosoma mansoni: an in vivo study of drug-induced autophagy in the gastrodermis. J Helminthol 58: 59-68.

Coelho PMZ, Katz N, Rocha MO, Souza CP, Mello RT 1988. Schistosoma mansoni: infected snails as a tool to screen antischistosomal drugs. Internl Parasitol 18: 167-170.
Coles GC 1979. The effect praziquantel on Schistosoma mansoni. J Helminthol 53: 31-33.

De Duve C, Wattiaux R 1966. Functions of lysosomes. Annu Rev Physiol 28: 435-492.

Doenhoff MJ, Kusel JR, Coles GC, Cioli D 2002. Resistance of Schistosoma mansoni to praziquantel: is there are problem? Trans R Soc Trop Med Hyg 96: 465-469.

Dietl P, Haller T, Wirleitner B, Friedrich F 1996. Two different store-operated $\mathrm{Ca} 2+$ entry pathways in MDCK cells. Cell Calcium 20: 11-19.

Gonnert R, Andrews P 1977. Praziquantel, a new broad-spectrum antischistosomal agent. $Z$ Parasitenkd 52: 129-150.

Jans R, Sartor M, Jadot M, Poumay Y 2004. Calcium entry into keratinocytes induces exocytosis of lysosomes. Arch Dermatol Res 296: 30-41.

Liang YS, Coles GC, Doenhoff MJ, Southgate VR 2001. In vitro responses of praziquantel-resistant and -susceptible Schistosoma mansoni to praziquantel. Int J Parasitol 31: 1227-1235.

Liang YS, Dai JR, Zhu YC, Coles GC, Doenhoff MJ 2003. Genetic analysis of praziquantel resistance in Schistosoma mansoni. Southeast Asian J Trop Med Public Health 34: 274-280.

McLaren DJ 1980. The adult worm. In KN Brown, Schistosoma mansoni: the Parasite Surface in Relation to Host Imunity, Research Studies Press, Chichester, p. 1-27.

Mehlhorn H, Becker B, Andrews P, Thomas H, Frenkel JK 1981. In vivo and in vitro experiments on the effects of praziquantel on Schistosoma mansoni. Arzneimitellforschung 31: 544-554.

Oliveira FA 2005. Avaliação do Efeito do Praziquantel, da Oxamniquina e da Associação dessas Duas Drogas sobre o Verme Adulto de Schistosoma mansoni, MSc Thesis, Fiocruz, Belo Horizonte, $82 \mathrm{pp}$.

Oliveira FA, Kusel JR, Ribeiro F, Coelho PMZ 2006. Responses of the surface membrane and excretory system of Schistosoma mansoni to damage and to treatment with praziquantel and other biomolecules. Parasitology 132: 321-330.

Pax RA, Bennett JL, Fetterer R 1978. A benzodiazepine derivative and praziquantel: effects on musculature of Schistosoma mansoni and Schistosoma japonicum. NaunynSchmiedebergs Arch Pharmacol 304: 309-315.

Pellegrino J, Katz N 1968. Experimental chemotherapy of Schistosoma mansoni. Adv Parasitol 6: 233-291.

Riley EM, Chappell LH 1990. Praziquantel treatment of Biomphalaria glabrata infected with Schistosoma mansoni - influence on snail fecundity. Parasitology 101: 211-217.

Smith JH, Chernin E 1974. Ultrastructure of young mother and daughter sporocysts of Schistosoma mansoni. J Parasitol 60: 85-89.

Touassem R, Combes C 1986. Comparative analysis of the Schistosoma mansoni and Schistosoma bovis production under influence praziquantel. Zeitschrift für Parasitenkunde 72: 345-351.

Yi XM, Combes C 1987. Effect of praziquantel on larval stages of Schistosoma japonicum. Trans R Soc Trop Med Hyg 81: 645-650. 
